\title{
Estatística de contagem com a plataforma Arduino
}

\author{
Counting statistics with the Arduino platform
}

\author{
A. M. Pereira ${ }^{1}$, A. C. F. Santos*1, H. S. Amorim ${ }^{1}$ \\ ${ }^{1}$ Instituto de Física, Universidade Federal do Rio de Janeiro, Rio de Janeiro, RJ, Brasil
}

\begin{abstract}
Recebido em 5 de abril de 2016. Revisado em 10 de maio de 2016. Aceito em 15 de maio de 2016
Descrevemos experimentos didáticos simples sobre detecção de radiação e estatística de contagens, utilizando a plataforma Arduino, para aplicação no ensino médio e superior. Os experimentos ilustram a natureza binomial do processo de decaimento radioativo. O aparato permite ao estudante medir as distribuições de Poisson e Gaussiana.
\end{abstract}

Palavras-chave: Arduino, estatística de contagens, radiação.

\begin{abstract}
We describe simple didactic experiments on radiation detection and statistics counts using the Arduino platform for application in secondary and higher education. The experiments illustrate the binominal nature of the radioactive decay process. The setup enables the students to measure the distributions of Poisson and Gauss.
\end{abstract}

Keywords: Arduino, counting statistics, radiation.

\section{Introdução}

Estatística de contagens é um assunto fascinante, embora muitas vezes negligenciado até mesmo nos cursos de graduação em Física. Vários são os exemplos de seu uso registrados na literatura: distribuições temporais de mortes por coices de cavalo, surtos de guerras, entre outros [1]. No presente trabalho, abordaremos um fenômeno estatístico presente em diversos setores da sociedade e tratado com superficialidade pelos meios de comunicação: decaimentos radioativos.

Uma questão que se levanta é: por que devemos ensinar radioatividade e sua natureza estatística para alunos de nível médio? Uma das razões consiste nas aplicações da natureza ionizante e estatística da radiação encontradas no cotidiano, como por exemplo, no diagnóstico e tratamento (Radioterapia) de doenças como o câncer e na irradiação de alimentos com o intuito de aumentar seu tempo de conservação. Outra razão consiste em auxiliar a compreensão adequada dos jovens e adultos sobre a polêmica que envolve os acidentes radioativos e a utilização da energia nuclear. Uma análise adequada

*Endereço de correspondência: toni@if.ufrj.br. destes assuntos requer conhecimentos básicos dos fenômenos envolvidos, da terminologia utilizada e capacidade de interpretar ideias científicas e suas aplicações em situações da vida real. É preciso que o aluno saiba diferenciar claramente radiação de material radioativo e processo de irradiação de contaminação. Acreditamos que estes objetivos não serão alcançados somente pela exposição do professor, mas através da participação ativa dos alunos, desafiando-os e oferecendo-lhes oportunidades de esclarecer suas concepções espontâneas.

Utilizaremos um contador Geiger-Müller [2], instrumento que permite medir intensidades de radioatividade (elementos radioativos e raios X). É um instrumento indispensável principalmente quando ocorrem acidentes radioativos, uma vez que identifica o nível de radiação de uma área em risco. Seu funcionamento consiste basicamente no seguinte: emissões radioativas atravessam a janela do tubo e passam por seu interior ionizando o gás - geralmente o argônio - contido a baixa pressão e um vapor orgânico ou um gás halogênio criando uma descarga elétrica. Esta se manifesta na forma de um pulso elétrico entre o eletrodo central (anodo) e o cilindro metálico (catodo) - onde a tensão aplicada 
é da ordem de $1 \mathrm{kV}$ - e é amplificado para um dispositivo acústico e/ou um contador, indicando que certa quantidade de energia foi absorvida pelo gás.

Este artigo tem dois objetivos: mostrar a natureza binomial dos processos de emissão e detecção da radiação e descrever experimentos que ilustram a utilização de um detector tipo Geiger-Muller de baixo custo, demonstrando as flutuações estatísticas em decaimentos radioativos.

\section{Aparato experimental}

Tendo em vista o elevado custo de um medidor Geiger comercial - inviáveis para aplicação em escolas públicas brasileiras -, escolhemos a plataforma open source Arduino [3-5] para o desenvolvimento de um projeto bem mais barato que pudesse medir satisfatoriamente a radiação em algumas situações. Nossa escolha baseou-se no fato do Arduino ser uma plataforma muito versátil de prototipagem eletrônica, de hardware livre, projetada com um microcontrolador Atmel AVR, com várias linhas de E/S digital e analógica e conexão USB. O software de programação (IDE) é livre, baseado numa linguagem simples, derivada essencialmente da linguagem $\mathrm{C} / \mathrm{C}++$. Uma das grandes vantagens é a disponibilidade de uma grande quantidade de bibliotecas de programas, de acesso livre, que usadas como sub-rotinas facilitam a comunicação com os mais diferentes tipos de sensores.

O tubo Geiger utilizado [6] é sensível à radiação beta e gama, detectando-as de forma multidirecional. Isto significa que a medição independe da orientação do sensor Geiger em relação à fonte de radioatividade, dependendo apenas da distância. A unidade de medida utilizada pelos tubos Geiger é basicamente o número de pulsos gerados num dado intervalo de tempo, isto é, "n"(um número inteiro) contagens por segundo (cps), ou contagens por minuto (cpm). Não é um valor de energia, mas apenas o número de pulsos.

Optamos em importar um kit pré-programado (Figura1), modular, que atendesse nossas necessidades. O módulo central é uma unidade de controle tipo Arduino (ATM328P), que incorpora circuitos complementares, um com a função de gerar uma tensão DC, ajustável na faixa aproximada de 100 $400 \mathrm{~V}$, para o acionamento do tubo Geiger e outro para possibilitar a conexão de um sensor GPS. Incluímos no projeto um modulo para armazenamento

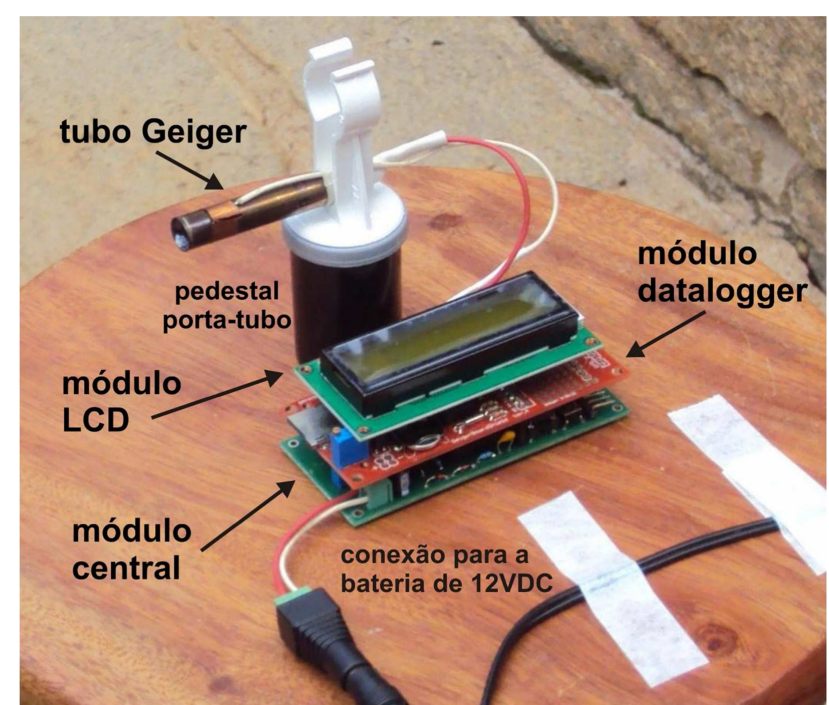

Figura 1: Montagem experimental. Vide texto para detaIhes.

de dados (datalogger) em cartão micro-SD e um display LCD para a visualização das medidas. O módulo datalogger incorpora um circuito integrado DS1307 que atua como um relógio de tempo real (RTC). O tubo Geiger é o modelo SBT9 $(6,5 \mathrm{~cm} \times$ $1 \mathrm{~cm})$ de fabricação russa. Toda a unidade é alimentada por uma bateria de $12 \mathrm{~V} / 12 \mathrm{Ah}$ que resulta numa autonomia de várias horas. Maiores detalhes podem ser encontrados na referência [7].

\section{Breve revisão dos modelos estatísticos}

Sob certas condições podemos predizer a função distribuição estatística, que descreverá os resultados de várias medições repetidas [8]. Podemos definir uma medida como uma contagem do número de sucessos resultando de um dado número de tentativas. Cada tentativa é um sucesso ou não. Para tudo que se segue, supomos que a probabilidade de sucesso é uma constante para todas as tentativas. Por exemplo, ao tentarmos observar um dado decaimento nuclear por um intervalo de tempo $t$, o número de tentativas é equivalente ao número de núcleos na amostra sob observação e a medida consiste em contar aqueles núcleos que decaem. Três modelos estatísticos específicos são introduzidos:

a) Distribuição Binomial. Este é o modelo mais geral e é largamente aplicável para todos os processos de probabilidade constante. Infelizmente, computacionalmente é muito trabalhosa para sistemas grandes, como por exem- 
plo, em decaimentos nucleares. Além disso, é pouco utilizada em aplicações nucleares.

b) Distribuição de Poisson. Este modelo é uma simplificação matemática da distribuição binomial com a condição da probabilidade de sucesso ser pequena. Em termos práticos, significa que o tempo de observação escolhido é pequeno comparado à meia-vida da fonte, ou que a eficiência de detecção é pequena.

c) Distribuição Gaussiana ou Normal. Este modelo é uma simplificação ainda maior no caso do número médio de sucessos ser relativamente grande (maior do que 20 ou 30). Esta condição se aplicará para qualquer situação na qual acumulamos mais do que algumas contagens durante a medida. Este é o caso mais frequente, de modo que o modelo Gaussiano é largamente aplicável a muitos problemas em estatística de contagem. Lembramos que os modelos acima tornam-se idênticos para processos com uma probabilidade individual de sucesso pequena, mas com um número grande de tentativas, de modo que o número médio esperado de sucessos é grande.

\subsection{A distribuição binomial}

Aqui alguma coisa é tentada repetidamente; a cada tentativa há sempre duas possibilidades, sucesso ou falha. Sendo pa probabilidade de sucesso e (1-p) a probabilidade de falha, podemos escrever

$$
\begin{aligned}
& 1=(1-p)+p \\
& 1^{N}=[(1-p)+p]^{N}=\sum_{n=0}^{N}\left(\begin{array}{c}
N \\
n
\end{array}\right) p^{n}(1-p)^{N-n}
\end{aligned}
$$

A distribuição binominal é a mais geral dos modelos estatísticos discutidos aqui. Se $N$ é o número de tentativas para o qual cada tentativa tem uma probabilidade de sucesso $p$, então a probabilidade de contar exatamente $x$ sucessos é dada por

$$
P(x)=\frac{N !}{(N-x) ! x !} p^{x}(1-p)^{N-x}
$$

onde $P(x)$ é a função probabilidade, como dada pela distribuição binomial e é definida somente para valores inteiros de $N$ e $x$. Algumas propriedades da distribuição binomial são importantes. Primeiramente, a distribuição é normalizada (vide equação (1)), ou seja

$$
\sum_{x=0}^{N} P(x)=1
$$

Também, sabemos que a média ou valor médio de uma distribuição é dada por

$$
\langle x\rangle=\sum_{x=0}^{N} x P(x)
$$

Se substituirmos a equação 2 para $P(x)$ na equação (4) e realizarmos a soma, encontramos o valor médio de $x$

$$
\langle x\rangle=p N
$$

O valor médio é, sem dúvidas, um valor fundamental para qualquer distribuição. É importante também obtermos um parâmetro que descreva a flutuação prevista por uma dada distribuição. Definimos agora a variância prevista $\sigma^{2}$, que é uma medida de como os dados estão distribuídos em torno da média prevista pelo modelo estatístico especifico $P(x)$,

$$
\sigma^{2} \equiv \sum_{x=0}^{N}(x-\langle x\rangle) P(x)
$$

Convencionalmente $\sigma^{2}$ é chamada de variância e enfatizamos o fato de que ela é associada com a distribuição de probabilidades prevista, por isto o nome variância prevista. É convenção definirmos também o desvio padrão como a raiz quadarada de $\sigma^{2}$. Agora, no caso da distribuição binomial, obtemos

$$
\begin{aligned}
& \sigma^{2}=N p(1-p) \\
& =\langle x\rangle(1-p) \\
& \sigma=\sqrt{\langle x\rangle(1-p)}
\end{aligned}
$$

Distribuição binomial para o decaimento radioativo

Poucos textos evidenciam que a radioatividade é essencialmente um processo binomialmente distribuído [8]. Considere o decaimento radioativo em um tempo tde um sistema contendo Nátomos radioativos. Estes $N$ átomos podem ser divididos em dois grupos: aqueles que decairão em um tempo te aqueles que não decairão. A partir da lei do decaimento exponencial para espécies radioativas, a probabilidade de que um dado átomo não decaia é $\mathrm{e}^{-\lambda t}$, onde $\lambda$ é a constante de decaimento para a espécie em questão. Então, a probabilidade ppara o decaimento é

Revista Brasileira de Ensino de Física, vol. 38, nº 4, e4501, 2016 


$$
p=1-e^{-\lambda t}
$$

A probabilidade $P(x)$ de que $x$ átomos decaiam em um intervalo de tempo $t$ é

$$
P(x)=\frac{N !}{(N-x) ! x !}\left(1-e^{-\lambda t}\right)^{x}\left(e^{-\lambda t}\right)^{N-x}
$$

O número verdadeiro de decaimentos no intervalo de tempo $t$ é

$$
\langle x\rangle=N\left(1-e^{-\lambda t}\right)
$$

e o desvio padrão fica

$$
\sigma=\sqrt{N\left(1-e^{-\lambda t}\right) e^{-\lambda t}}=\sqrt{\langle x\rangle e^{-\lambda t}}
$$

Para $\lambda \mathrm{t} \ll 1$, ou seja, para tempos de observação curtos comparados com a meia vida, o desvio padrão é simplesmente

$$
\sigma=\sqrt{\langle x\rangle}
$$

\subsection{A distribuição de Poisson}

Várias categorias de processos binários podem ser caracterizadas por uma baixa probabilidade de sucesso para cada tentativa individual. Nestes casos, a aproximação $p \rightarrow 0$ e $N \rightarrow \infty$ (na prática $N \geqslant 30$ e $p \leqslant 0,05)$, de tal modo que a média $\langle x\rangle=N p$ permaneça finita, será válida e algumas simplificações matemáticas podem ser aplicadas à distribuição binomial. Usando que

$$
\frac{N !}{(N-x) !} \approx N^{x}
$$

e

$$
(1-p)^{N-x} \approx e^{-p N}
$$

a distribuição binominal se reduz a

$$
P(x)=\frac{(p N)^{x} e^{-p N}}{x !}
$$

Assim como a distribuição binomial, a distribuição de Poisson é discreta. Ela essencialmente descreve processos para os quais a probabilidade de tentativa única é muito pequena, mas o número de tentativas é grande. Note que a distribuição binomial possui dois parâmetros: o número de tentativas $N$ e a probabilidade individual de sucesso $p$. Mas, podemos ver que apenas um parâmetro é necessário na distribuição de Poisson, o produto $N p$. Esta é uma simplificação muito útil porque precisamos conhecer apenas o valor médio da distribuição para reconstruir sua amplitude $P(x)$ para todos os outros valores do argumento $x$. Algumas das propriedades da distribuição binomial, também são válidas para o caso da distribuição de Poisson. A variância no caso da distribuição de Poisson é dada por

$$
\sigma^{2}=\langle x\rangle
$$

Enfatizamos que, em geral, os livros didáticos partem da equação $\frac{d N}{d t}=-\lambda N$ para o decaimento radioativo, e afirmam de forma ad hoc que o decaimento segue uma distribuição de Poisson, sem explicitar a sua natureza binomial.

\subsection{A distribuição Gaussiana}

Consideremos agora uma outra simplificação em que o valor médio da distribuição é alto (maior do que 20). Pode-se mostrar que esta simplificação adicional, utilizando a aproximação de Stirling

$$
x ! \cong \sqrt{2 \pi x}\left(\frac{x}{e}\right)^{x}
$$

leva à distribuição Gaussiana ou distribuição normal

$$
P(x)=\frac{1}{\sqrt{2 \pi\langle x\rangle}} e^{-\frac{(x-\langle x\rangle)^{2}}{2\langle x\rangle}}
$$

onde a equação 16 permanece válida.

A distribuição Gaussiana (também conhecida como distribuição normal) reside nos fundamentos da maioria dos métodos estatísticos em ciências. Os testes $\chi^{2}, \mathrm{~F}$, Student, e outros, se baseiam na hipótese de que a quantidade em consideração segue uma distribuição Gaussiana. Além disso, flutuações Gaussianas (ou ruído Gaussiano) aparecem em quase todas as medidas em Física.

\section{Resultados}

Nesta seção, descrevemos sugestões de práticas utilizando o aparato descrito anteriormente.

\subsection{Prática 1: medindo a radiação de fundo.}

Inicialmente, sugerimos que o professor comente sobre a estrutura da montagem experimental e desenvolva a ideia de que existe uma radiação de fundo presente em todos os locais do planeta. O medidor 
Geiger deve ser apresentado aos alunos explicando sua finalidade, seu princípio de funcionamento e como é feita sua montagem (esquema técnico). Assim que o medidor é ligado, são ouvidos cliques e observados dados sendo impressos no visor de LCD. Explicamos que esta experiência nos proporciona coletar contagens de radiação, onde cada pulso elétrico que foi detectado corresponde a uma partícula detectada e o valor oferecido no visor é a média estatística destas leituras. Com isso temos a oportunidade de discutir a fonte destes cliques e porque esses cliques posteriormente tornam-se uma contagem de radiação. Pelo fato da estrutura do nosso aparato experimental medir eventos de colisão e ionização da matéria as medidas encontradas não seguem um padrão de repetição. Por isso, é imprescindível montar um histograma como forma de representar essa dispersão de dados e determinarmos o valor médio e desvio padrão correspondentes (é preciso articular com os alunos um conjunto de ideias e conceitos que fazem parte dessa experiência simples).

Nessa prática, cada medida corresponde ao número de contagens observadas em 1 minuto. Tendo em vista a dispersão dos dados, repetimos a medida 92 vezes. Os dados foram medidos sequencialmente e registrados automaticamente no cartão de memória. Os dados foram transportados para o computador e construímos o histograma mostrado na Figura 2. Os dados foram agrupados em classes (intervalos) automaticamente pelo software Origin2015 [9] para a adequada análise de dispersão. Ressaltamos

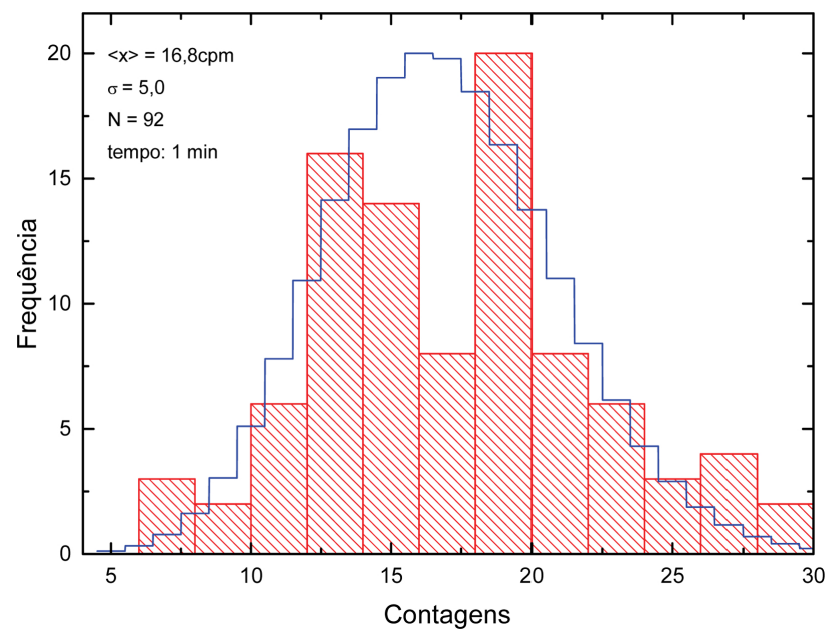

Figura 2: Histograma de medidas da radiação ambiente. Barras verticais: dados experimentais; curva cheia: ajuste usando a distribuição de Poisson, equação (15), com $\langle x\rangle$ $=16,8 \mathrm{cpm}, N=92$ e $\sigma=5,0 \mathrm{cpm}$. a importância do professor de Física na tarefa de capacitar os alunos em analisar dados através de um histograma de medidas e justificar o seu uso.

Sendo o processo de medida um processo estatístico, nos interessa então encontrar para a radiação local um "valor médio" (linha cheia do gráfico) e seu desvio padrão (linhas pontilhadas indicando os limites laterais). Como resultado, o software nos fornece $(16,8 \pm 5,0) \mathrm{cpm}$, que denominamos radiação ambiente ou radiação de fundo.

\subsection{Prática 2: medindo a radiação de uma amostra mineral radioativa.}

Em seguida, temos como objetivos determinar a influência de uma fonte radioativa, de baixo teor, utilizando o medidor Geiger e uma amostra mineral de monazita. A monazita é um fosfato que contem em sua composição o elemento radioativo Tório. A amostra utilizada consiste de grãos de monazita dispersa em areia (areia monazítica), previamente extraída de uma praia de Guarapari/ES. A areia monazítica foi acondicionada num vidro (vidro de azeitonas tamanho médio). A fonte assim constituída pode ser considerada extensa quando comparamos suas dimensões com os valores envolvidos nas práticas realizadas. As medidas foram feitas com o tubo Geiger encostado no vidro e as contagens obtidas armazenadas em um intervalo de tempo de $1 \mathrm{~min}$, conforme mostra a Figura 3.

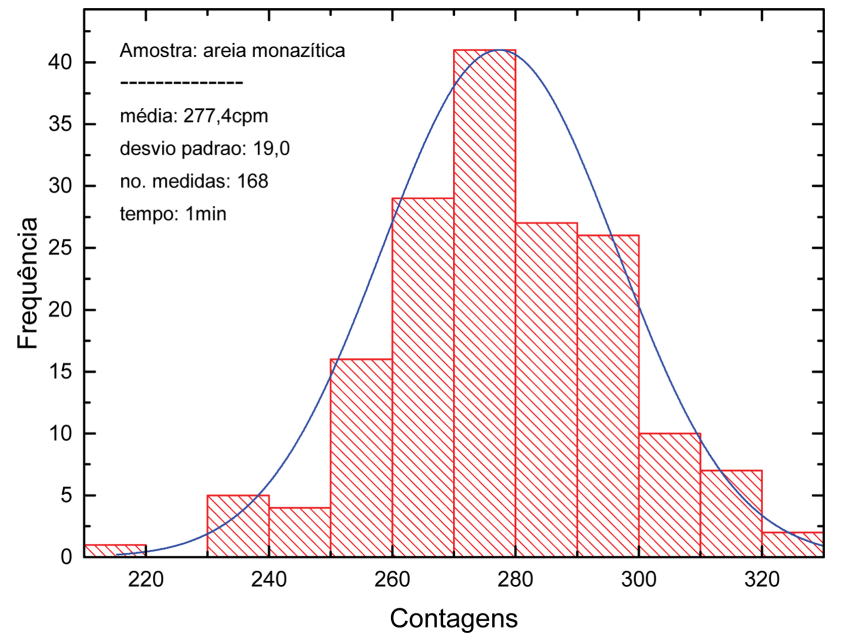

Figura 3: Histograma de medidas da radiação da areia monazítica. Barras verticais: dados experimentais; curva cheia: ajuste utilizando a distribuição normal, equação (16). Note que utilizamos aqui a distribuição normal apenas para fins ilustrativos. Poderíamos também ajustar utilizando a distribuição de Poisson. 
O valor médio encontrado para a leitura da radiação na presença da areia monazítica foi de 277,4 cpm, o que significa um aumento de $260,6 \mathrm{cpm}$ em relação a radiação de fundo. Com isso, a radiação nas proximidades do medidor ficou 15,5 vezes maior. Experimentos deste tipo nos ajudam a medir a influência das fontes radioativas nas medidas de radiação e sua investigação em situações cotidianas. Nesta ocasião, o professor pode aproveitar para discutir a validade do ajuste utilizando a distribuição normal.

\subsection{Prática 3: medindo a intensidade radiação em função da distância à fonte radioativa.}

A próxima prática consiste em mostrar a dependência com a distância à fonte nas leituras de radiação ao afastarmos gradualmente o medidor da fonte radioativa. Para tanto, além do medidor Geiger e do vidro com areia monazítica, utilizamos também uma régua. Ainda na presença da fonte radioativa, vamos agora variar a distância entre o medidor e a fonte e verificar qual a relação existente entre o valor médio de radiação e a distância da fonte até o medidor. Para isso, afastamos radialmente o tubo Geiger $2,0 \mathrm{~cm}$ e armazenamos valores de contagens durante 1,0 min. Várias medidas são tomadas sequencialmente para se caracterizar uma média e um desvio padrão. Em seguida, afastando o medidor mais $2,0 \mathrm{~cm}$ repetimos o processo de contagem, e assim sucessivamente até o mesmo atingir uma distância de $20,0 \mathrm{~cm}$ da fonte. Os pontos destacados na Figura 4 representam os valores médios calculados com o respectivo desvio padrão em função de sua posição. Os histogramas das medidas encontram-se no apêndice A. A linha cheia serve apenas para guiar os olhos.

Verificamos um decaimento nas medidas até uma distância de aproximadamente $10 \mathrm{~cm}$, e após este valor, os valores oscilam em torno de $90 \mathrm{cpm}$. Sabemos que a dependência da intensidade de radiação detectada com a distância à fonte depende de vários fatores, entre eles o fato de nossa fonte não ser pontual, a diminuição do ângulo sólido de detecção e o retroespalhamento por objetos da vizinhança. A discussão desses efeitos está além do escopo deste artigo.

\subsection{Prática 4: absorção da radiação pela matéria.}

Outras práticas podem ser introduzidas como, por exemplo, observar o efeito de blindagem da radiação através do uso de variados absorvedores, especificando o poder de penetração de cada partícula envolvida. Uma sugestão interessante é relacionar a absorção com a espessura do absorvedor (no caso, alumínio) e verificar a aplicabilidade da Lei de Beer-Lambert [10]. Para esta prática, precisaremos além do medidor Geiger, areia monazítica e papel alumínio de uso doméstico. Aumentamos gradualmente o número de folhas de alumínio interpostas

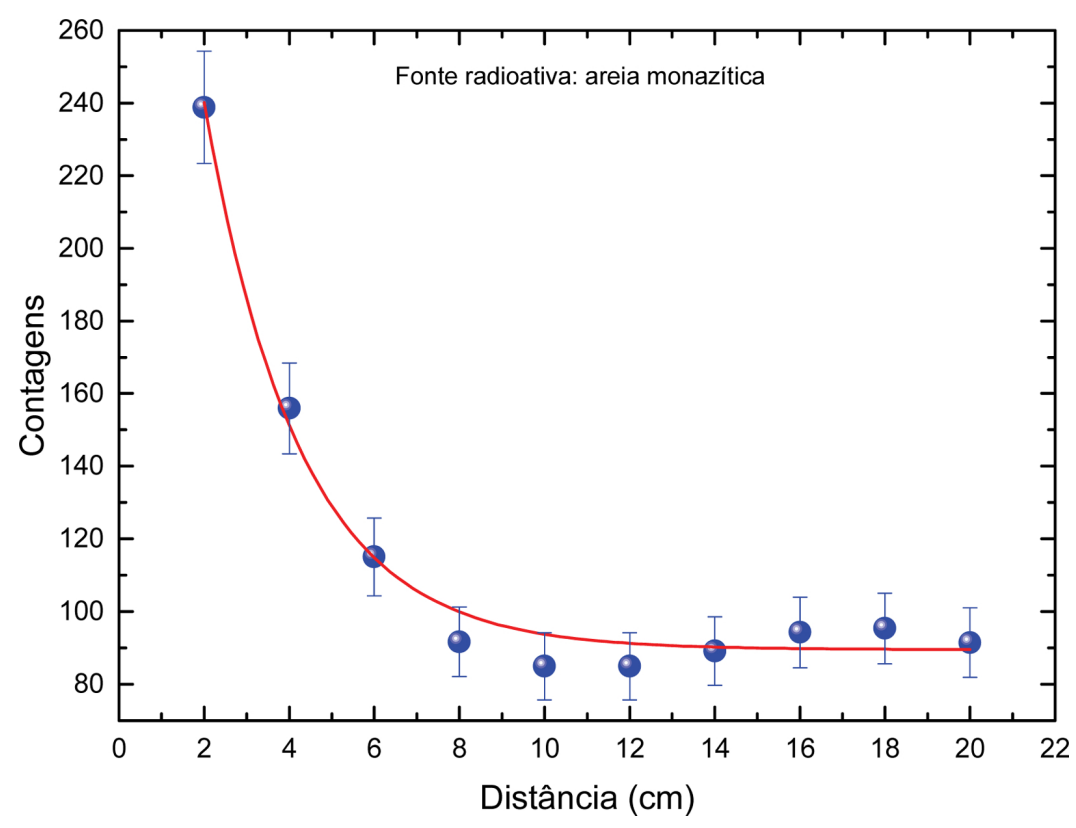

Figura 4: Relação entre a leitura da radiação e a distância à fonte. 
entre a fonte radioativa e o tubo Geiger, mantidos em posições fixas e verificamos qual o efeito de atenuação. As incertezas apresentadas na Figura 5 representam os valores médios calculados com seus respectivos desvios padrão em função do número de folhas utilizadas. Com o aumento da espessura (número de folhas (n)) de alumínio, verificamos que a leitura do medidor tem um decaimento aproximadamente exponencial. A linha cheia que pode ser observada na Figura 4 foi obtida ajustando-se pelo método dos mínimos quadrados a função,

$$
y(\text { contagem })=y_{0}+A \cdot e^{-b . n}
$$

Em seguida o professor pode buscar relacionar a radiação com a altitude e encontrar soluções satisfatórias para medir a radiação cósmica secundária (abaixo do "chuveiro cósmico"). Fora do espaço físico da sala de aula, esta prática tem como proposta identificar a influência da altitude na radiação cósmica secundária que incide em determinada região. Para diminuir a influência terrestre algumas soluções são conhecidas, tais como: uma espessa camada com mais de $1 \mathrm{~m}$ de gelo [9] e o uso de um balão atmosférico - ou durante um voo de avião - para medir a radiação em altitudes elevadas, distantes do solo. Desenvolvemos o experimento com o objetivo de buscar uma analogia entre a absorção atmosférica e a quantidade de andares de um prédio. Neste experimento, temos a tarefa de relacionar a absorção atmosférica da radiação cósmica secundária com o efeito de blindagem oferecido pelos andares (lajes) de um prédio. O local escolhido foi o bloco A do prédio do Centro de Tecnologia da UFRJ. Deixa-

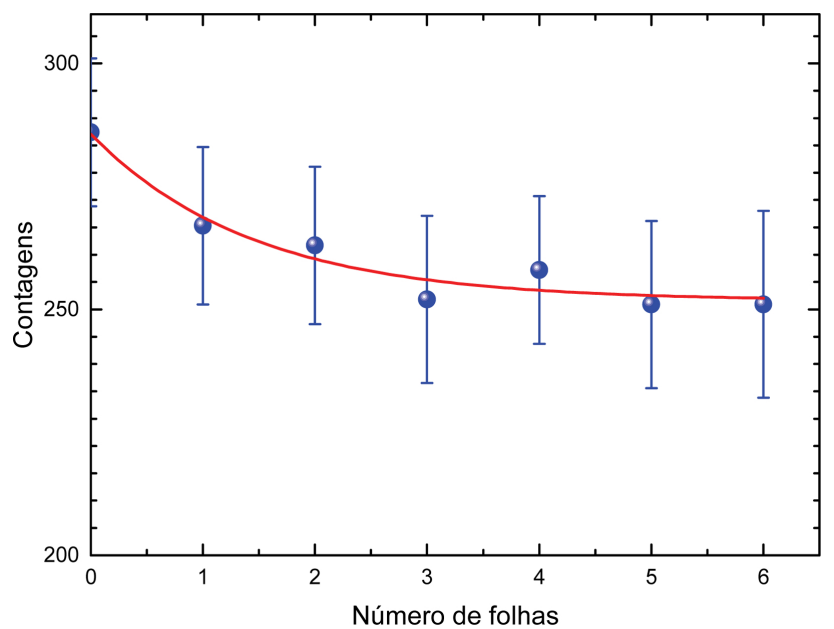

Figura 5: Relação entre a leitura de radiação e o número de folhas de alumínio. mos o medidor no $2^{\circ}, 4^{\circ}$ e $6^{\circ}$ andares coletando dados durante um intervalo de tempo de $30 \mathrm{~min}$ em cada andar. $\mathrm{O}$ valor médio e o respectivo desvio padrão para cada andar encontram-se representados na Figura 6.

Como era de se esperar [11], o efeito de blindagem diminui conforme subimos os andares do prédio uma vez que a quantidade de lajes de concreto acima de nós diminui, atenuando sutilmente a absorção da radiação cósmica secundária e aumentando a radiação de fundo medida: $(19,6 \pm 5,0) \mathrm{cpm}$ no $2^{\circ}$ andar, $(20,5 \pm 4,3) \mathrm{cpm}$ no $4^{\circ}$ andar e $(22,1 \pm$ $4,4) \mathrm{cpm}$ no $6^{\circ}$ andar. Em linhas gerais, podemos relacionar a blindagem oferecida por grandes aumentos de altitude (diminuição da coluna de ar) com a diminuição da quantidade de concreto conforme subimos os andares de um prédio. Sabemos que emissões de radiação vindas das paredes (areia com tório) influenciam as medidas, mas consideramos a proposta viável na medida em que essa influência é praticamente constante. Observamos uma tendência condizente com o que é fisicamente esperado, nos encorajando a colocar como uma proposta para os estudantes. Acreditamos que medições em prédios mais altos com uma amostragem maior (maior tempo de coleta por andar) diminuiriam o desvio padrão e evidenciariam o efeito procurado. No nosso caso, verificamos uma diferença do valor médio das contagens de aproximadamente $13 \%$ entre o andar mais baixo $\left(2^{\circ}\right.$ andar $)$ e o andar mais alto ( $6^{\circ}$ andar). Aqui vale uma discussão adicional com os alunos sobre incerteza de uma medida. Embora os resultados sejam, a princípio, condizentes com o

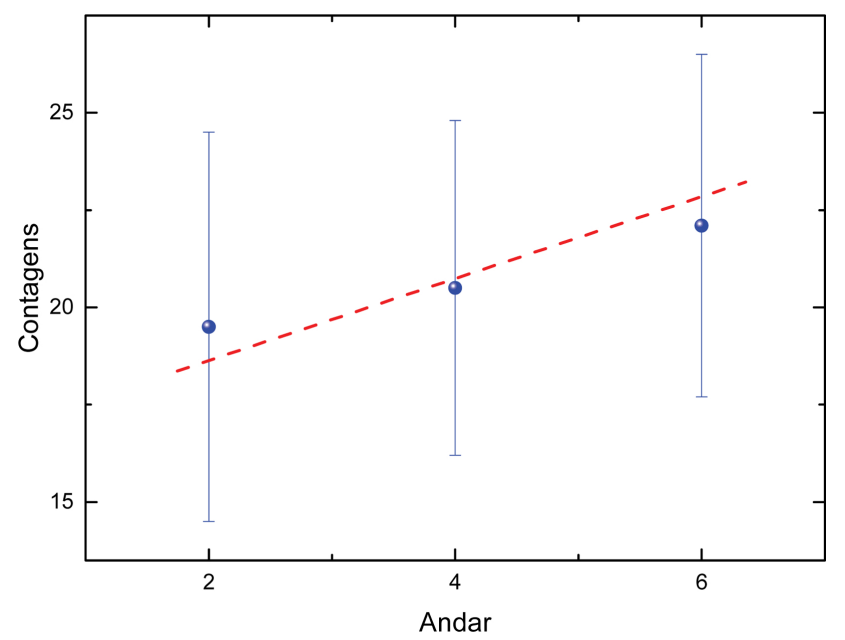

Figura 6: Número de contagens no detector Geiger em função do andar. 
que é fisicamente esperado, as incertezas associadas às medidas não permitem afirmar que de fato ocorre uma blindagem.

\section{Conclusões}

Neste artigo apresentamos experimentos simples sobre detecção de radiação e estatística de contagens utilizando uma plataforma Arduino com medidor Geiger acoplado para utilização no ensino médio e superior. Através destes experimentos, ilustramos a natureza binomial do processo de decaimento radioativo, permitindo ao estudante medir as distribuições de Poisson e Gaussiana. Acreditamos estar introduzindo uma metodologia capaz de ultrapassar os muros da sala de aula, aproximando o ensino de Física à vida real e de grande relevância e potencial para a relação ensino-aprendizagem assim como para o desenvolvimento científico nacional. Esta mudança na forma de abordar e ensinar o assunto Física das Radiações facilita a compreensão do processo de emissão e detecção de radiação, contribuindo desta forma com a alfabetização científica, onde o indivíduo consegue transpor o conhecimento aprendido para a vida social.

\section{Referências}

[1] S.K. Chakarvarti, Nand Lal e K.K. Nagpaul, European Journal of Physics 1, 133 (1980).

[2] E.M. Kakuno, Revista Brasileira de Ensino de Física 36, 1315 (2014).

[3] H. Cordova e A.C. Tort, Revista Brasileira de Ensino de Física, aceito para publicação.

[4] H.S. Amorim, M.A. Dias e V. Soares, Revista Brasileira de Ensino de Física 37, 4310, (2015).

[5] L.R.M. Carvalho e H.S. Amorim, Revista Brasileira de Ensino de Física 36, 3501, (2014).

[6] Encontrado em https://sites.google.com/ site/diygeigercounter/home

[7] Programa de Pós-Graduação em Ensino de Física, Instituto de Física, UFRJ: http://www.if.ufrj.br/ pef/producao_ academica/dissertacoes/2014_Alexandre_ Pereira/material_instrucional_Alexandre_ Pereira.pdf

[8] L. Ruby, Am. J. Phys. 45, 380 (1977).

[9] http://www.originlab.com/ (versão 2015).

[10] A.C.F. Santos, S.D. Magalhaes e N.V.C. Faria, Nucl. Instr. Meth. Phys. Res. B 261, 264 (2007).

[11] B. Jones The Phys. Teach. 31, 458 (1993). 